\title{
AVALIAÇÃO DO PERFIL E QUALIDADE DE VIDA DO ACADÊMICO DE ENFERMAGEM
}

\author{
PROFILE EVALUATION AND QUALITY OF LIFE OF NURSING SCHOLARS
}

\author{
Silas Alves Machado ${ }^{a^{*}}$, Gleidson Brandão Oselame ${ }^{b^{*}}$, Eduardo Borba Neves ${ }^{c^{* *}}$ \\ asilasalvesmachado@yahoo.com, bgoselame@ics.curitiba.pr.gov.br, cborbaneves@hotmail.com \\ *Centro Universitário Campos de Andrade - Curitiba (PR), Brasil \\ **Universidade Tecnológica Federal do Paraná (UTFPR) - Curitiba (PR), Brasil
}

Data de recebimento do artigo: 10/06/2015

Data de aceite do artigo: 05/10/2015

\section{RESUMO}

Introduçáo: Pesquisas envolvendo a avaliação da qualidade de vida de acadêmicos de enfermagem admitem concluir que a qualidade de vida dessa população é negativa em relação à saúde física, psíquica e mental. Objetivo: Avaliar o perfil socioeconômico e a qualidade de vida de acadêmicos de enfermagem de uma instituição de ensino superior na cidade de Curitiba, Paraná. Métodos: Foram utilizados um questionário para avaliação socioeconômica com 30 perguntas fechadas e o questionário de avaliação da qualidade de vida, denominado WHOQOL-bref, da Organização Mundial da Saúde. Os dados socioeconômicos foram analisados por estatística descritiva. Os dados da avaliação da qualidade de vida foram submetidos ao cálculo das médias e desvio-padrão dos domínios das respostas utilizando-se um software desenvolvido em Excel em que se calcula o escore "total" do entrevistado que realiza a média aritmética simples dos escores das 26 questóes do instrumento. Resultados: Compuseram a amostra deste estudo 156 acadêmicos de enfermagem, sendo 78 de cada turno (manhã e noite). A faixa etária predominante no turno da manhã foi de 18 a 20 anos (38,46\%; n=30), mesma faixa etária que predominou no turno da noite $(47,44 \% ; n=37)$. O sexo feminino representou a maioria dos acadêmicos em ambos os turnos, respectivamente $88,46 \%(n=69)$ e 79,49\% $(n=62)$. Em relação à avaliação da qualidade de vida, o domínio com menor média foi o relativo ao meio ambiente (13,03\%). Relativo às facetas que compuseram os domínios, dor e desconforto, dependência de medicação ou de tratamentos e sentimentos negativos obtiveram médias mais baixas. Conclusão: Sugere-se a elaboração de estratégias de suporte para os acadêmicos de enfermagem no enfrentamento das adversidades impostas pela rotina acadêmica.

Palavras-chave: Escolas de enfermagem; estudantes de enfermagem; qualidade de vida.

\section{ABSTRACT}

Introduction: Researches involving the evaluation of the quality of life of nursing scholars admit to concluding that the quality of life of this population is negative in relation to physical, psychological, and mental health. Objective: To evaluate the socioeconomic profile and quality of life of nursing scholars of a higher education institution in the city of Curitiba, Paraná, Brazil. Methods: A questionnaire for socioeconomic evaluation with 30 closed questions and the World Health Organization's questionnaire for assessing quality of life (WHOQOL-bref) were used. Socioeconomic data were analyzed using descriptive statistics. Data from the assessment of quality of life were submitted to the mean and standard deviation calculation of the answers' areas using a software developed in Excel that calculates the "total" score of the interviewee and performs the simple arithmetic average of the scores of the instrument's 26 questions. Results: The sample of this study was composed of 156 nursing scholars, 78 of each shift (morning and evening). The predominant age group in the morning shift was $18-20$ years $(38.46 \% ; n=30)$, same age group that prevailed on the night shift $(47.44 \%, \mathrm{n}=37)$. Females accounted for most scholars in both shifts, respectively $88.46 \%(\mathrm{n}=69)$ and $79.49 \%(n=62)$. Regarding the evaluation of quality of life, the domain with the lowest average was the Environment (13.03\%). On the facets that made up the domains, pain and discomfort, dependence on medication or treatments, and negative feelings obtained the lowest averages. Conclusion: The preparation of support strategies for nursing scholars in facing adversities imposed by the academic routine is suggested.

Keywords: Nursing schools; nursing students; quality of life. 


\section{Introdução}

Nas últimas três décadas houve um aumento quantitativo no ensino superior no Brasil, seja em faculdades, cursos, ou em matrículas facilitando o ingresso em instituiçóes públicas e privadas. No entanto, o nível social do aluno interpóe a procura dos cursos $^{1}$.

Nesse sentido, as transformaçóes que vêm ocorrendo em instituiçóes de ensino superior de enfermagem demonstram esses efeitos. Pesquisas foram realizadas a fim de projetar o perfil e estimar quanto vale a colaboração da verdadeira educação para a carreira profissional ${ }^{2}$. Com essas mudanças, há necessidade de conhecer o perfil socioeconômico e cultural das pessoas que desejam fazer uma graduação em enfermagem ${ }^{3}$.

$O$ perfil do acadêmico de enfermagem tem incentivado alguns autores ${ }^{4,5}$ a estudá-lo. Destaca-se que a maioria desse perfil é composta por pessoas jovens de 18 a 22 anos de idade, do sexo feminino, que moram com seus pais, são casadas, sem filhos e com renda própria trabalhando na área da saúde.

Nesse perfil é comum que acadêmicos de enfermagem passem por vários comportamentos em relaçáo a sua qualidade de vida ${ }^{6,7}$. As instituiçóes devem se preocupar com a qualidade de vida de seus futuros profissionais, pois durante a graduaçáo os acadêmicos estão sujeitos a isolamentos, dificuldades de aprendizagem, drogas e doenças neurológicas ${ }^{8,9}$.

Algumas pesquisas de qualidade de vida realizadas com acadêmicos de enfermagem admitem concluir que sua qualidade de vida é negativa em relação à saúde física, psíquica e mental e que não estão prontos para a enfermagem por falta de prática em resolver problemas em momentos difíceis. Muitas vezes a falta de qualidade de vida está vinculada ao abandono familiar, baixa de autoestima, ausência de descanso, liberdade, atenção e exercício físico ${ }^{10}$.

Nesse contexto, o acadêmico de enfermagem deve ser visto como um ser humano que optou por cuidar, conviver com limitaçóes e até a morte. Portanto, depara com situaçôes de sofrimento que podem contribuir tanto para a humanização desse processo ou sua banalização. Ressalta-se ainda que a exposição do acadêmico de enfermagem seja a mesma de muitos profissionais no mercado de trabalho, sendo considerado um fator crucial no comprometimento da qualidade de vida ${ }^{11}$.

Estudo realizado em seis cursos de enfermagem na regiâo Sul do Brasil indicou que 36\% dos acadêmicos de enfermagem apresentam problemas significativos referentes à qualidade de vida ${ }^{12}$.
Dessa forma, o objetivo deste estudo foi avaliar o perfil socioeconômico e qualidade de vida de acadêmicos de enfermagem de uma instituição de ensino superior na cidade de Curitiba, Paraná.

\section{Metodologia}

Tratou-se de um estudo descritivo transversal com análise quantitativa dos dados. A coleta de dados se deu em dois momentos distintos: no primeiro momento aplicou-se um questionário socioeconômico com 30 questôes para acadêmicos do primeiro ao oitavo período do curso de enfermagem. No segundo momento foi aplicado o questionário WHOQOLBref que avalia a qualidade de vida, desenvolvido pela Organização Mundial da Saúde (OMS), composto por 26 questóes.

Os critérios de inclusão foram acadêmicos de enfermagem com idade superior a 18 anos, regularmente matriculados e que consentiram sua participação por meio do Termo de Consentimento Livre e Esclarecido (TCLE).

Para o procedimento de cálculo das médias e desvio-padrão dos domínios das respostas utilizou-se o software desenvolvido por Pedroso, Pilatti, Gutierrez e Picinin ${ }^{13} \mathrm{em}$ Excel que calcula o escore "total" do entrevistado e realiza a média aritmética simples dos escores das 26 questôes do instrumento.

Foram respeitados os preceitos éticos conforme determinam as Diretrizes e Normas Regulamentadoras de Pesquisa envolvendo Seres Humanos, por meio da Resolução 466/2012 do Conselho Nacional de Saúde. Este estudo foi aprovado pelo Comitê de Ética em Pesquisa do Centro Universitário Campos de Andrade por meio do parecer n. 969.785/2015.

\section{Resultados}

\section{Perfil socioeconômico dos acadêmicos de enfermagem}

Compuseram a amostra 156 acadêmicos de enfermagem, sendo 78 de cada turno. A faixa etária predominante no turno da manhã foi de 18 a 20 anos $(38,46 \% ; n=30)$, mesma faixa etária que predominou no turno da noite $(47,44 \% ; n=37)$. O sexo feminino representou a maioria dos acadêmicos em ambos os turnos respectivamente $(88,46 \% ; n=69)$ e $(79,49 \% ; \mathrm{n}=62)$. As demais variáveis são apresentadas na Tabela 1 . 
Tabela 1: Perfil socioeconômico dos acadêmicos de enfermagem. Curitiba, Paraná, Brasil, 2015.

\begin{tabular}{|c|c|c|c|c|}
\hline \multirow{2}{*}{ Faixa etária } & $\mathbf{n}$ & $\%$ & $\mathbf{n}$ & $\%$ \\
\hline & \multicolumn{2}{|c|}{ Manhã } & \multicolumn{2}{|c|}{ Noite } \\
\hline $18-20$ anos & 30 & 38,46 & 37 & 47,44 \\
\hline 25-29 anos & 8 & 10,26 & 10 & 12,82 \\
\hline 30-39 anos & 26 & 33,33 & 13 & 16,67 \\
\hline Mais de 39 anos & 14 & 17,95 & 18 & 23,08 \\
\hline \multicolumn{5}{|l|}{ Sexo } \\
\hline Feminino & 69 & 88,46 & 62 & 79,49 \\
\hline Masculino & 9 & 11,54 & 16 & 20,51 \\
\hline \multicolumn{5}{|l|}{ Estado civil } \\
\hline Solteiro(a) & 38 & 48,72 & 31 & 39,74 \\
\hline Casado(a) & 21 & 26,92 & 33 & 42,31 \\
\hline Viúvo(a) & 1 & 1,28 & - & - \\
\hline União estável & 18 & 23,08 & 14 & 17,95 \\
\hline \multicolumn{5}{|l|}{ Meio de transporte } \\
\hline Carro & 19 & 24,36 & 35 & 44,87 \\
\hline Moto & 6 & 7,69 & 1 & 1,28 \\
\hline Ônibus & 49 & 62,82 & 23 & 29,49 \\
\hline Outros & 4 & 5,13 & 19 & 24,36 \\
\hline \multicolumn{5}{|l|}{ Renda mensal } \\
\hline Menos de um salário-mínimo & 6 & 7,69 & 9 & 11,54 \\
\hline Um a dois salários-mínimos & 42 & 53,85 & 22 & 28,21 \\
\hline Dois a três salários-mínimos & 22 & 28,21 & 29 & 37,18 \\
\hline Três a cinco salários-mínimos & 6 & 7,69 & 16 & 20,51 \\
\hline Acima de cinco salários-mínimos & 2 & 2,56 & 2 & 2,56 \\
\hline \multicolumn{5}{|l|}{ Composiçáo da renda } \\
\hline O próprio(a) & 26 & 33,33 & 28 & 35,90 \\
\hline Cônjuge ou companheiro(a) & 25 & 32,05 & 30 & 38,46 \\
\hline Pais & 18 & 23,08 & 18 & 23,08 \\
\hline Outras pessoas & 9 & 11,54 & 2 & 2,56 \\
\hline \multicolumn{5}{|l|}{ Vida econômica } \\
\hline Não trabalha e seus gastos são custeados & 12 & 15,38 & 10 & 12,82 \\
\hline Trabalha e é independente financeiramente & 25 & 32,05 & 27 & 34,62 \\
\hline Trabalha, mas não é independente financeiramente & 21 & 26,92 & 23 & 29,49 \\
\hline Trabalha e é responsável pelo sustento da família & 20 & 25,64 & 18 & 23,08 \\
\hline \multicolumn{5}{|l|}{ Local de moradia } \\
\hline Urbana & 76 & 97,44 & 70 & 89,74 \\
\hline Rural & 2 & 2,56 & 8 & 10,26 \\
\hline \multicolumn{5}{|l|}{ Local de trabalho } \\
\hline Comércio & 10 & 12,82 & 10 & 12,82 \\
\hline Indústria & 1 & 1,28 & 1 & 1,28 \\
\hline Saúde & 54 & 69,23 & 55 & 70,51 \\
\hline Não trabalha & 13 & 16,67 & 12 & 15,38 \\
\hline \multicolumn{5}{|l|}{ Cargo que ocupa } \\
\hline Chefe & 1 & 1,28 & 6 & 7,69 \\
\hline Gerente & 1 & 1,28 & 1 & 1,28 \\
\hline Gestor & 5 & 6,41 & 3 & 3,85 \\
\hline Encarregado & 4 & 5,13 & 2 & 2,56 \\
\hline Técnico de enfermagem & 49 & 62,82 & 41 & 52,56 \\
\hline Não trabalha & 13 & 16,67 & 12 & 15,38 \\
\hline Outros & 5 & 6,41 & 13 & 16,67 \\
\hline
\end{tabular}




\section{Avaliação da qualidade de vida}

Em relação à avaliação da qualidade de vida, o domínio com menor média foi o meio ambiente $(13,03 \%)$. As demais variações dos domínios são apresentadas na Tabela 2.
Relativo às facetas que compuseram os domínios, dor e desconforto, dependência de medicação ou de tratamentos e sentimentos negativos obtiveram médias mais baixas. A Figura 1 apresenta estas e as demais variáveis analisadas.

Tabela 2: Percepção dos acadêmicos de enfermagem quanto à qualidade de vida segundo os domínios. Curitiba, Paraná, Brasil, 2015.

\begin{tabular}{|c|c|c|c|c|c|c|}
\hline Domínio & Média & Desvio-padráo & Coeficiente de variação & $\begin{array}{c}\text { Valor } \\
\text { mínimo }\end{array}$ & $\begin{array}{c}\text { Valor } \\
\text { máximo }\end{array}$ & Amplitude \\
\hline Físico & 14,78 & 2,40 & 16,24 & 9,14 & 20,00 & 10,86 \\
\hline Psicológico & 14,91 & 2,26 & 15,17 & 9,33 & 20,00 & 10,67 \\
\hline Relações sociais & 15,09 & 3,03 & 20,07 & 6,67 & 20,00 & 13,33 \\
\hline Meio ambiente & 13,03 & 2,19 & 16,84 & 7,50 & 19,50 & 12,00 \\
\hline Autoavaliação da QV & 14,71 & 2,78 & 18,91 & 6,00 & 20,00 & 14,00 \\
\hline TOTAL & 14,30 & 1,90 & 13,31 & 10,15 & 19,54 & 9,38 \\
\hline
\end{tabular}

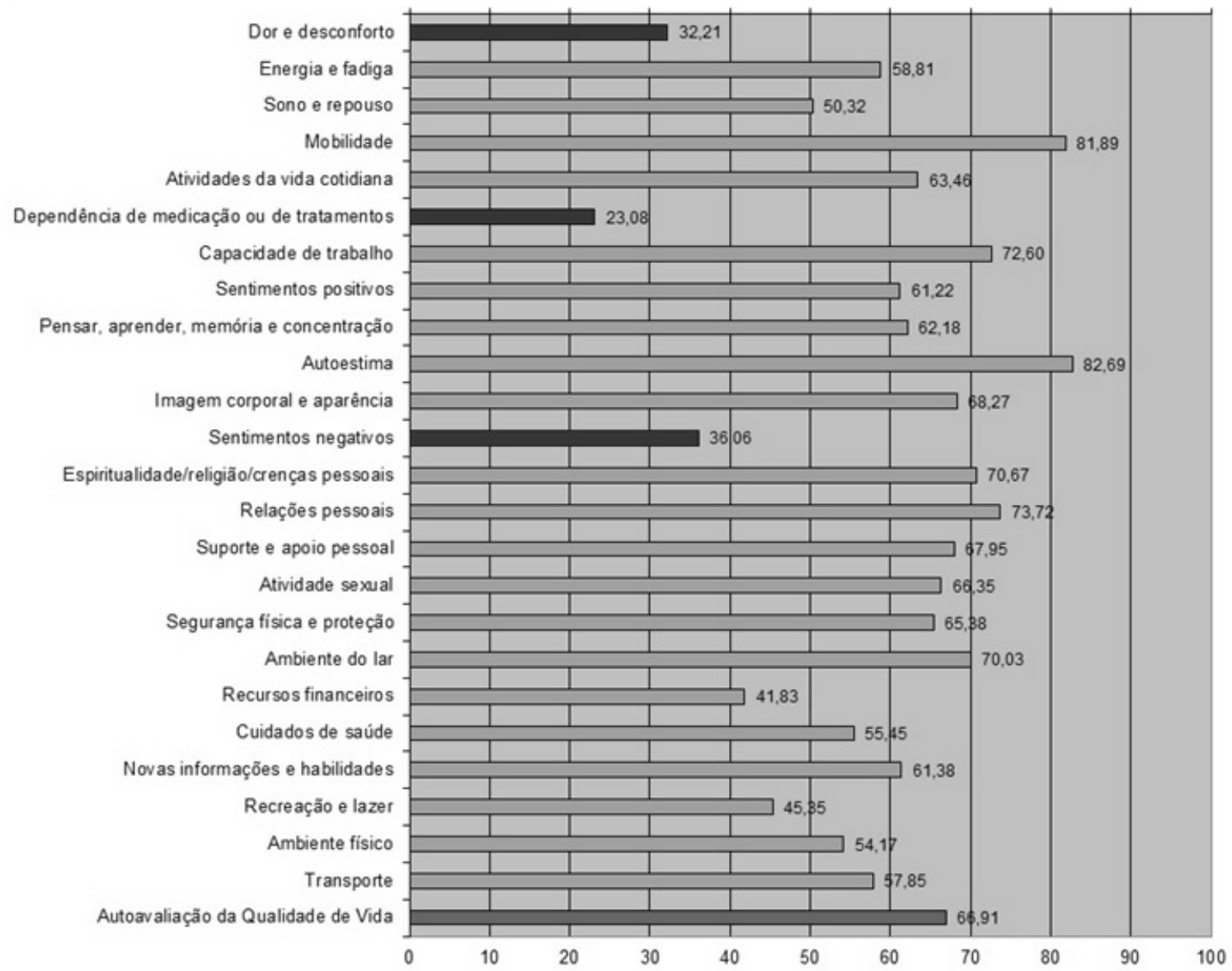

Figura 1: Facetas da avaliação da qualidade de vida dos acadêmicos de enfermagem.

\section{Discussão}

A faixa etária predominante em ambos os turnos respectivamente foi de 18 a 20 anos $(38,46 \%$ n $=30$; $47,44 \% \mathrm{n}=37)$. O perfil do acadêmico de enfermagem é formado por jovens que estão aptos a entrar para o mercado de trabalho. Destaca-se que este é um fator positivo à medida que os jovens profissionais obterão oportunidades mais cedo, no que se refere ao crescimento e progresso. Isso mostra que eles estão interessados em estudar e trabalhar ${ }^{14}$.

Dados semelhantes emergiram do estudo proposto por Oliveira, Mininel e Feli ${ }^{11}$ realizado com 65 acadêmicos de enfermagem, em que a população apresentou 
idade entre 22-24 anos, sendo a maioria mulheres solteiras. Neste estudo o perfil também foi concordante com o estudo citado, em que predominou a população do sexo feminino em ambos os turnos respectivamente $(88,46 \% n=69 ; 79,49 \% \mathrm{n}=62)$.

Nesse sentido, nos cursos de enfermagem a presença feminina se mostra sempre superior a masculina. $\mathrm{O}$ aumento do sexo feminino nos cursos de graduação de enfermagem se deve aos conhecimentos anteriores da enfermagem e ao relacionamento humano pelo fato da mulher ser mais carismática ${ }^{5}$. Estudos demonstram a predominância de mulheres nos cursos de enfermagem, com percentuais variando de 89 a 97,8\%5,6,15.

Relativo à renda, os acadêmicos de enfermagem do período da noite apresentaram renda de um a dois salários-mínimos $(53,85 \% ; n=42)$ inferior a renda declarada pela maioria dos acadêmicos do turno da noite, com renda de dois a três salários-mínimos $(37,18 ; \mathrm{n}=29)$. Essa diferença no poder aquisitivo pode ter influenciado pela escolha do meio de transporte utilizado até a instituição de ensino, em que $62,82 \%(\mathrm{n}=49)$ utilizam o ônibus no período noturno e $44,87 \%(n=35)$ utilizam o carro no período da manhá.

Um estudo semelhante realizado no estado do Paraná em uma universidade pública objetivou avaliar a qualidade de vida de acadêmicos de graduaçáo em enfermagem buscando correlacionar as variáveis sociodemográficas de acadêmicos do $1^{\circ}$ e $4^{\circ}$ ano do curso. Foram avaliados 34 acadêmicos do primeiro ano $(89,5 \%)$ e 33 acadêmicos do quarto ano (100\%). O instrumento utilizado para avaliar a qualidade de vida foi o questionário WHOQOL-Bref. Predominaram no estudo mulheres, jovens, solteiras, com renda per capita média familiar mensal de mais de um salário-mínimo ${ }^{16}$.

Outra variável analisada se refere ao tipo de ocupação dos acadêmicos de enfermagem. Em ambos os turnos prevaleceram trabalhadores da área da saúde, sobretudo, técnicos de enfermagem, com $62,82 \%(n=49)$ no turno da noite e 52,56\% (n=41) pela manhá. Ressalta-se que a busca dos técnicos de enfermagem pela graduação está associada com alguns fatores, tais como ascensão profissional, busca pelo conhecimento científico e mudança de papel dentro da equipe de enfermagem ${ }^{17}$.

Esse fenômeno apresenta impacto direto em faculdades privadas que oferecem a graduação em enfermagem, sobretudo pela oferta de bolsas de estudo e unidades na periferia das grandes cidades facilitando o acesso dos estudantes ${ }^{17}$.

Em relação à avaliação da qualidade de vida, o domínio com menor média foi o meio ambiente $(13,03)$ e com maior média relações sociais $(15,09)$. Em um estudo semelhante, o maior escore médio de qualidade de vida foi para o domínio físico $(13,1)$, e o menor para o domínio meio ambiente $(12,7)^{16}$.
Dados de um estudo realizado com seis cursos de enfermagem, localizados na regiáo Sul do Brasil, cujo objetivo foi avaliar a qualidade de vida de acadêmicos de enfermagem com o uso do WHOQOL-Bref, mostraram que $64 \%$ apresentaram satisfaçáo com sua qualidade de vida. No entanto, 36\% apresentaram problemas significativos, que apontam para a necessidade da implantação de programas de apoio e enfrentamento das situaçôes de sofrimento ${ }^{12}$.

Outro estudo realizado com 264 estudantes de enfermagem utilizou como instrumento o Quality of Life Index (QLI). Os resultados encontrados de forma geral foram positivos, próximos do valor máximo do score (30), sendo encontradas médias de 25,17 para o domínio saúde/funcionamento; 25,24 para o socioeconômico; 25,91 para o psicológico/espiritual; 25,66 para o domínio família e 25,40 para o QLI total. O que chama atenção é que sofrem alteraçôes nos diferentes anos que compóem o curso, sendo 26,16 para o $1^{\circ}$ ano; 24,48 para o $2^{\circ}$ ano; 25,46 para o $3^{\circ}$ ano e 25,23 para o $4^{\circ}$ ano, refletindo que os estudantes do $1^{\circ}$ ano possuem melhor qualidade de vida, com queda do

QLI do $1^{\circ}$ para o $2^{\circ}$ que apresentaram os piores índices de qualidade de vida, depois recuperados nos $3^{\circ} \mathrm{e}$ $4^{\circ}$ anos $^{6}$.

Os baixos escores do domínio meio ambiente geram preocupaçáo, pois o cotidiano agitado e a valorização apenas do futuro geram certa desvalorização do presente e sugerem sacrifícios em prol dos objetivos, o que pode levar a um distanciamento das coisas que dão prazer ao acadêmico ${ }^{7}$. Alguns fatores associados para a baixa qualidade de vida se referem à falta de acolhimento por parte dos professores e enfermeiros de campo, falta de integração com equipe e alunos de outros cursos e a carga horária excessiva para o aluno trabalhador ${ }^{8}$.

Relativo às facetas que compuseram os domínios, dor e desconforto, dependência de medicação ou de tratamentos e sentimentos negativos obtiveram médias mais baixas. Dessa forma, ressalta-se a busca por situaçóes promotoras de qualidade de vida no ambiente acadêmico. Podem ser citadas atividades com este perfil as experiências extracurriculares, relação professor/aluno e o relacionamento entre alunos ${ }^{8}$.

Destaca-se que os acadêmicos têm diferentes percepçóes sobre qualidade de vida. Alguns fatores favorecem e outros comprometem a qualidade de vida durante o período de graduação, e podem configurar o perfil de saúde e doença, associado especialmente ao estresse ${ }^{11}$.

Portanto, a formação do enfermeiro voltada ao desempenho técnico específico não pode mais ser aceita pelas instituições de ensino superior, necessitando voltar-se para a formaçáo do profissional cidadão, com competência técnica e científica, e, sobretudo, com ampla visão da dimensão humana ${ }^{18}$. 


\section{Conclusão}

A pesquisa apresentou a predominância do sexo feminino com faixa etária de 18 a 20 anos. A qualidade de vida apresentou média de $14,30( \pm 1,90)$. A maior média entre os domínios foi as relaçóes sociais $(15,09 \pm 3,03)$ e a menor média o domínio meio ambiente $(13,03 \pm 2,19)$. Ainda, chamam atenção sentimentos negativos, dependência de medicação e dor e desconforto, presentes em grande parte dos acadêmicos de enfermagem. Dessa forma, esses resultados podem resultar em barreiras à boa atuação profissional dos futuros profissionais de saúde. Dessa maneira, sugere-se a elaboração de estratégias de suporte para os acadêmicos de enfermagem no enfrentamento das adversidades impostas pela rotina acadêmica.

\section{Referências}

1. Martins CB. O ensino superior brasileiro nos anos 1990. São Paulo em Perspectiva. 2000;14(1):41-60.

2. Henriques RLM, Clos AC. Desafios da graduação em enfermagem: a primeira geração de enfermeiros do novo currículo. Rev Enferm UERJ. 2000;8(2):71-2.

3. Sanches MO, Pedro ENR. Ações e expressóes de cuidado na prática educativa de enfermeiros docentes. Rev Gaú Enfermagem. 2008;29(1):11.

4. Arcuri E, Araújo TL, Oliveira M. Fatores que influenciaram alunos ingressantes na Escola de Enfermagem da USP, em 1981, na escolha da enfermagem como opção profissional. Rev Esc Enferm USP. 1983;17(1):5-16.

5. Santos CE, Leite MMJ. O perfil do aluno ingressante em uma universidade particular da cidade de Sáo Paulo. Rev Bras Enfermagem. 2006;59(2):154-6.

6. Kawakame PMG, Miyadahira AMK. Qualidade de vida de estudantes de graduação em enfermagem. Rev Esc Enferm USP. 2005;39(2):164-72.

7. Beuter M, Alvim NAT, Mostardeiro S. O lazer na vida de acadêmicos de enfermagem no contexto do cuidado de si para o cuidado do outro. Texto \& Contexto Enferm. 2005;14(2):222-8.

8. Oliveira RA, Ciampone MHT. A universidade como espaço promotor de qualidade de vida: vivência e expressóes dos alunos de enfermagem. Texto \& Contexto Enferm. 2006;15(2):254-61.

9. Jorge MSB, Rodrigues ARF. Serviços de apoio ao estudante oferecidos pelas escolas de enfermagem no Brasil. Rev Latino-Enfermagem. 1995;3(2):59-68.

10. Geib LTC, Saupe R. Educare: ensaiando a pedagogia do cuidado. Texto \& Contexto Enferm. 2000;9(2, pt. 2):497-508.

11. Oliveira BM, Mininel VA, Felli VEA. Qualidade de vida de graduandos de enfermagem. Rev Bras Enferm. 2011;64(1):130-5.

12. Saupe R, Nietche EA, Cestari ME, Giorgi MDM, Krahl M. Qualidade de vida dos acadêmicos de enfermagem. Rev Latino-Am Enfermagem. 2004;12(4):636-42.

13. Pedroso B, Pilatti LA, Gutierrez GL, Picinin CT. Cálculo dos escores e estatística descritiva do WHOQOL-Bref através do Microsoft Excel. Rev Bras Qualidade de Vida. 2010;2(1):31-6.

14. Pizzoli LML. Qualidade de vida no trabalho: um estudo de caso das enfermeiras do Hospital Heliópolis. Ciênc Saúde Coletiva. 2005;10(4):1055-62.

15. Wetterich NC, Melo MRAC. Sociodemographic profile of undergraduate nursing students. Rev Latino-Am Enfermagem. 2007;15(3):404-10.

16. Eurich RB, Kluthcovsky A. Avaliação da qualidade de vida de acadêmicos de graduaçáo em enfermagem do primeiro e quarto anos: influência das variáveis sociodemográficas. Rev Psiquiatr RS. 2008;30(3):211.

17. Medina NVJ, Takahashi RT. A busca da graduação em enfermagem como opçáo dos técnicos e auxiliares de enfermagem. Rev Esc Enferm USP. 2003;37(4):101-8.

18. Oliveira Camillo S, Silva AL, Nascimento AJ. Percepçôes do graduando de enfermagem sobre a dimensão humana no seu aprendizado. Rev Latino-Am Enfermagem. 2007;15(2):207-13.

\section{Como citar este artigo:}

Machado AS, Oselame GB, Neves EB. Avaliação do perfil e qualidade de vida do acadêmico de enfermagem. Rev. Aten. Saúde. 2016;14(47): 55-60. 